\title{
RAYMOND TRÉMOLIÈRES
}

\section{Le problème des roulements de quarts pour les entreprises à feu continu}

Revue française d'automatique, d'informatique et de recherche opérationnelle. Recherche opérationnelle, tome 10, nº V1 (1976), p. 71-101.

<http://www.numdam.org/item?id=RO_1976_10_1_71_0>

(C) AFCET, 1976, tous droits réservés.

L'accès aux archives de la revue « Revue française d'automatique, d'informatique et de recherche opérationnelle. Recherche opérationnelle » implique l'accord avec les conditions générales d'utilisation (http://www.numdam.org/ legal.php). Toute utilisation commerciale ou impression systématique est constitutive d'une infraction pénale. Toute copie ou impression de ce fichier doit contenir la présente mention de copyright.

\section{Numdam}

Article numérisé dans le cadre du programme

Numérisation de documents anciens mathématiques

http://www.numdam.org/ 


\title{
LE PROBLEMME DES ROULEMENTS DE QUARTS POUR LES ENTREPRISES A FEU CONTINU ( $\left.{ }^{*}\right)\left({ }^{1}\right)$
}

\author{
par Raymond TrÉMOLIÈRes $\left({ }^{2}\right)$
}

\begin{abstract}
RÉSUMÉ. - L'étude concerne la génération automatique de plannings de roulements de quarts en monovalence. On en donne les fondements algorithmiques et on établit un théorème permettant de reconnaître si deux plannings cycliques sont redondants l'un par rapport à l'autre. Dans une dernière partie on a reporté quelques plannings obtenus par ordinateur.
\end{abstract}

\section{PRÉSENTATION}

On aborde ici le problème de la génération automatique de cycles de roulements de quarts. L'étude débouche sur la définition de problèmes mathématiques nouveaux auxquels nous attribuons le vocable de " combinatoire cyclique ». De façon précise nous n'étudierons ici qu'une partie de ce nouveau domaine de recherche, nous attachant principalement à la résolution des roulements de quarts en monovalence.

\section{INTRODUCTION}

("Vous savez reconnaître si une combinaison est correcte; la belle affaire si vous ne possédez pas l'art de choisir entre toutes les combinaisons possibles. ")

Henri PoInCARÉ, Science et Méthode, p. 137.

Les roulements de quarts intéressent aujourd'hui un nombre accru d'usines ou de services - pétroles, chimie, sidérurgie, centrales électriques, mines, rextiles, industrie du papier, hôpitaux, permanences de tous ordres -, et il est assez curieux de noter que la littérature à grande diffusion soit pratiquement muette sur ce sujet $\left({ }^{3}\right)$. A en croire nos contemporains, les usines seraient de grands déserts dès la nuit tombée, et quant aux services fonctionnant jour et nuit, il y aurait quelques rares individus ayant consacré leur vie à une existence purement nocturne!

En fait le travail par roulement apparaît dès que le coût encouru par l'arrêt d'une production devient prohibitif (arrêter un four peut demander plusieurs

(*) Reçu avril 1975.

( $\left.{ }^{1}\right)$ Cette étude a été réalisée pour la Compagnie Rhénane de Raffinage de Reichstett (Alsace).

( ${ }^{2}$ ) Maître de Conférence à l'Université de Droit, d'Économie et des Sciences d'AixMarseille, I.A.E., Aix-en-Provence.

$\left({ }^{3}\right) C f$. Bibliographie en fin de cette note.

Revue Française d'Automatique, Informatique et Recherche Opérationnelle $\mathrm{n}^{\circ}$ février 1976. 
mois de remise en route) et de façon générale dès qu'il s'agit d'un service ou d'un travail dont la durée dépasse une certaine limite tolérable pour l'nndividu.

Dans cette étude nos nous limiterons aux roulements de quarts, terminologie que l'on attache généralement aux situations dans lesquelles une disponibilité de 24 heures sur 24 est requise.

Dans ce domaine, la mise en place et la gestion - car il s'agit véritablement d'une " gestion " - du travail par roulement n'est pas chose aisée. Les responsables le savent bien, pour passer des heures à résoudre les difficultés que posent:

- l'harmonisation du travail et la satisfaction des désirs de chacun ( ${ }^{1}$ );

- la législation : taux de tıavail hebdomadaire fixe mais pouvant varier dans le temps;

- les nouvelles lois sur la formation continue : dégager des heures ou des périodes à cet effet;

- la volonté de diversifier le travail en rendant possible le changement périodique à cet effet;

- les divers congés auxquels les ouvriers ont droit;

- ainsi que les absences dont il faut tenir compte sans trop perturber le planning général.

Outre ces contraintes il en est d'autres, physiologiques ou psychologiques que l'on ne saurait négliger.

Bien qu'il mériterait largement que soient étudiées toutes les implications, médicales, psychologiques, sociales, juridiques et économiques du travail par roulement $\left({ }^{2}\right)$ notre intention est de nous en tenir plus particulièrement à l'aspect opérationnel du problème : compte tenu d'un certain nombre de contraintes, comment générer automatiquement des plannings cycliques acceptables afin de déterminer ceux qui offrent le plus de " fiabilité " pour pallier certaines contraintes occasionnelles comme les congés, la formation ou les absences.

\section{CE QU'EST UN ROULEMENT DE QUARTS EN $3 \times 8$}

Dans une raffinerie de pétrole le cracking, la distillation, le traitement, les mélanges sont des opérations automatiques qui demandent une surveillance continue de 24 heures sur 24. Pour fixer les idées, il y a à Reichstett 25 opérations de surveillance à assurer de façon continue. On parle alors de 25 postes de travail à chacun desquels doit se trouver en permanence un opérateur.

( ${ }^{1}$ Souvent renforcés par la pression des syndicats.

$\left({ }^{2}\right)$ On parle encore de «travail posté ».

Revue Française d'Automatique et Recherche Opérationnelle 


\section{Cycles, tours et quarts}

L'être humain ayant ses limites, on convient de faire succéder 3 opérateurs $\left({ }^{1}\right)$ sur un même poste dans une période de 24 heures :

- 1 opérateur de nuit $(N)$, généralement de $21 \mathrm{~h}$ à $5 \mathrm{~h}$;

- 1 opérateur de soir $(S)$, généralement de $13 \mathrm{~h}$ à $21 \mathrm{~h}$;

- 1 opérateur du matin $(M)$, généralement de $5 \mathrm{~h}$ à $13 \mathrm{~h}$.

Chaque opérateur doit assurer une permanence de 8 heures consécutives - on parle alors des $3 \times 8$. Sans autre contrainte que celle de la continuité, il faudrait donc 3 opérateurs par poste et au total $25 \times 3=75$ opérateurs pour faire tourner la raffinerie. Une telle hypothèse est évidemment irrecevable du fait que chaque ouvrier travaillerait ainsi $7 \times 8$ heures $=56$ heures par semaine. Outre ceci, les opérateurs (qui n'aiment pas tellement les " nuits ») se mettent généralement d'accord pour prendre à tour de rôle les quarts [si l'on veut, au sens de " quartiers " $\left({ }^{2}\right)$ ] de nuit, de soir ou de matin. Habituellement, on adopte l'ordre de changement $N, S, M ; N, S, M$, etc. On parle alors de roulements de quarts et le schéma type de roulement - dans une hypothèse de 56 heures de travail - se présente comme suit :

\begin{tabular}{|c||l|l|l||l|l|}
\hline operateurs & L M M J V & L M M J V & L M M J V & L M M J V & L M M J V \\
\hline$A \rightarrow$ & N N N N N N & S S S S S S S & M M M M M M & N N N N N N & S S S S S S S \\
\hline$B \rightarrow$ & MM M M MM & N N N N N N & S S S S S S S & M M & \\
\hline$C \rightarrow$ & S S S S S S S & M M M M M M & N N N NNN N & S & \\
\hline
\end{tabular}

Figure 1.

Roulement de quarts de 56 heures, cycle de 3 semaines.

On reconnaît ici la notion de cycle de quarts, suite qui se succède indéfiniment et identique à elle-même. La longueur d'un cycle de quarts peut se mesurer en heures, jours, semaines, ou mois. Dans l'exemple précédent le cycle est de 3 semaines mais un cycle peut être plus court : à la limite il pourrait être de 3 jours (insoutenable pour l'organisme) offrant la configuration suivante :

\begin{tabular}{|c|l|}
\hline opérateurs & L M M J V . . \\
\hline $\mathrm{A} \longrightarrow$ & $\longrightarrow$ N M N S M \\
\hline $\mathrm{B} \longrightarrow$ & M N S M N \\
\hline $\mathrm{C} \longrightarrow$ & S M N S M N \\
\hline
\end{tabular}

Figure 2.

Roulement de quarts de 56 heures, cycle de 3 jours.

( $\left.{ }^{1}\right)$ Ceci donne une charge de 8 heures pour chaque opérateur; mais l'on peut convenir de faire se succéder 4 opérateurs (donc un travail de 6 heures) ou même plus : c'est ce qui est le cas par exemple dans les mines de charbon ou le trajet pour aller de la surface au fond peut demander 1 heure (donc 2 heures pour l'aller et retour).

$\left.{ }^{2}\right)$ La terminologie « roulements de quarts » est un vieux terme de marine : officier de « quart », prendre son « quart », etc.

n février 1976. 
Un point de vocabulaire : dans un cycle nous appelons tour une suite ininterrompue soit de nuits, soit de soirs, soit 'de matins, soit de repos de durée d'au moins 24 heures, ... La longueur d'un tour $(N, S$ ou $M)$ est le nombre de quarts consécutifs soit de nuit, soit de soir, soit de matin.

Nous nous permettrons aussi de parler du début d'un cycle bien que ceci n'ait guère de sens vu la nature cyclique du planning. Un début de cycle ne sera donc qu'une convention visant à faciliter l'exposé. Nous admettons qu'un cycle débute toujours par le premier quart d'un tour de nuit.

\section{Les journées normales}

Outre les tours de nuit $(N)$, de soir $(S)$, de matin $(M)$, ou de repos $(-)$, les plannings de $3 \times 8$ comportent généralement - et nous verrons pourquoi des tours de jours $(J)$ pendant lesquels les ouvriers sont affectés à d'autres travaux (entretien, formation, disponibilité pour des remplacements éventuels, ...). A titre d'illustration, le planning de roulement de 40 heures, pratiqué à Reichstett est le suivant.

\begin{tabular}{|c|c|c|c|c|c|c|}
\hline \multicolumn{2}{|c|}{ opérateurs } & LMM J V & $L M M J V$ & L M M J V & $L M M J V$ & LMM J V \\
\hline A & $\longrightarrow$ & NNNNNNN & $\because$ s s s s & $S \mathrm{~S} \cdot \mathrm{MMMM}$ & MMM . . & J J J J \\
\hline B & $\longrightarrow$ & J J J J ... & NN NNN N N & $-s \mathrm{~s} s \mathrm{~s}$ & $S S-M M M M$ & MM M. \\
\hline $\mathrm{c}$ & $\longrightarrow$ & M M M .... & J J J J & N N N N N N N & - s s s s s & $S \mathrm{~S}-\mathrm{MMMM}$ \\
\hline D & $\longrightarrow$ & S S - M M M M & MMM . . & $\mathrm{J} \mathrm{J} \mathrm{J} \mathrm{J} \cdots$ & N N N N N N & . s s s s s \\
\hline $\mathrm{E}$ & $\longrightarrow$ & $\ldots$ s s s s & SS.MMMM & MMM $\cdots$ & J J J J. & NNNNNN N \\
\hline
\end{tabular}

Figure 3.

Roulement de quarts standard de $\mathbf{4 0}$ heures (Reichstett), cycle de $\mathbf{5}$ semaines.

Le lecteur remarquera que les roulements de quarts présentent une configuration « en escalier » bien particulière.

\section{Le passage des consignes}

Dans certaines compagnies on tient compte dans le calcul du taux de travail du passage des consignes : un opérateur ayant terminé son quart (par exemple de matin) reste quelques minutes supplémentaires pour renseigner celui qui lui succède (en quart de soir) sur les divers incidents qui ont pu se produire et sur quoi il convient peut-être de fixer plus son attention, et puis, pourquoi pas, pour bavarder de tout et de rien sur le travail. A Ato-Chimie de Marseille le temps de passage des consignes est compté à $7 \mathrm{mn} 37 \mathrm{~s}$ ce qui fait en ce qui les concerne un chiffre rond de 16 heures de passation de consignes (deux " quarts ») dans leur cycle.

Dans d'autres compagnies, le temps de passage des consignes n'est pas décompté et il donne droit à prendre des repos en lieu et place de certaines journées normales que l'ouvrier choisit à sa guise. 
Pour la généralité on pourrait considérer que le temps de passage des consignes est plus grand lorsqu'un opérateur reprend un nouveau tour (de $N$, de $S$ ou de $M$ ). Nous n'envisagerons cependant pas ce cas ici, d'ailleurs il ne semble pas que ceci soit considéré dans la réalité.

\section{Les problèmes concernant l'établissement et la gestion des plannings cycliques}

Les problèmes soulevés par les roulements de quarts sont très nombreux.

Parmi ceux à caractère " mathématique » on peut retenir assez grossièrement les suivants :

Un nombrc d'ouvriers sur un poste étant donné, quels sont (s'ils en existent) les plannings pour lesquels :

- on réalise un taux hebdomadaire moyen de travail [par exemple 40 heures par semaine $\left.\left({ }^{1}\right)\right]$;

- la durée du passage des consignes est comprise dans un certain intervalle donné a priori;

- il y a un nombre " maximal » de week-end;

- la longueur des tours de nuits, de soirs, ou de matin est comprise entre certaines bornées données a priori;

- on dégage des périodes suffisamment « longues » de journées normales;

- on dégage des périodes suffisamment " longues » de journées de repos:

- le nombre de jours de repos après ou avant certains (ou tous les) tours est plus grand qu'une certaine valeur.

C'est essentiellement sur ces points que va porter notre étude.

\section{MISE EN FORME DU MODẼLE}

\section{3 a. Hypothèses}

Sans trop nous éloigner de la généralité nous adopterons, pour ce qui est des roulements de quarts en monovalence, les hypothèses suivantes :

- hypothèses sur les postes

$1^{\circ}$ A chaque poste est affecté un nombre fixé d'opérateurs - généralement 5 ou 6 - qui y sont attachés exclusivernent.

$2^{\circ}$ Sur un poste il doit y avoir en permanence, c'est-à-dire 24 heures sur 24 , un opérateur et un seul.

( $\left.{ }^{1}\right)$ Loi du 21 juin 1936.

no février 1976. 


\section{- hypothèses sur les cycles}

$3^{\circ}$ Sur un poste les opérateurs ont tous des mêmes cycles, la seule chose qui puisse différer est leur début dans le temps; cependant ce sera toujours un même jour dans la semaine - le lundi pour fixer les idées. Un cycle est nécessairement de longueur fini.

Des trois hypothèses précédentes on déduit sans difficulté les constatations suivantes :

- un cycle aura toujours pour longueur un multiple de semaines - ceci se justifiant par le fait que deux cycles successifs doivent avoir évidemment les mêmes caractéristiques, par exemple pour ce qui est de la disposition des week-ends;

- un cycle comporte un même nombre de nuits, de soirs et de matins;

- en monovalence, la longueur d'un cycle (en jours ou en semaines) est un multiple du nombre d'opérateurs $\left({ }^{1}\right)$;

- le nombre de nuits, de soirs ou de matins est égal à la longueur en jours du cycle divisé par le nombre d'ouvriers travaillant sur le poste : c'est alors évidemment un multiple de 7.

- hypothèses sur les tours de $N, S, M$

$4^{\circ}$ Dans un cycle les tours doivent se succéder dans un ordre donné qui à Reichstett est $N \rightarrow S \rightarrow M\left({ }^{2}\right)$; ainsi, un tour de matin ne peut reprendre avant qu'un tour de $N$ et un tour de $S$ n'aient été effectués. La longueur des tours de $N, S$ et $M$ est comprise dans un intervalle $[a, b]$ avec $a \geqq 1$, $b \leqq 7\left({ }^{3}\right)$.

\section{- hypothèses sur les tours de journées normales $(J)$}

$5^{\circ}$ Les tours de jours $(J)$ peuvent s'intercaler n'importe où entre les tours de $N, S$ et $M$ - mais l'habitude est de les intercaler entre les tours de $M$ et ceux de $N$. Normalement les quarts de $J$ ne peuvent se situer un samedi ou un dimanche, d'où, pour la longueur des tours. de jours, des bornes $\left[a_{J} b_{J},\right]$ avec $a_{J} \geqq 0, b_{J} \leqq 5$.

$6^{\circ}$ D'un tour à l'autre il y a d'habitude au moins 1 jour de repos (4); notant $\left(\alpha_{N}, \alpha_{s}, \alpha_{M}, \alpha_{J}\right)$ et $\left(\omega_{N}, \omega_{S}, \omega_{M}, \omega_{J}\right)$ respectivement le nombre minimum

(1) Nous en laissons la démonstration rigoureuse à la sagacité du lecteur. Pour le mettre sur la voie on remarquera que pour une période donnée dont la longueur $L$ est celle d'un cycle, on retrouve évidemment $L$ nuits dans le planning. Or sur cette période chaque opérateur doit avoir la même " charge " en nuits, d'où le résultat...

${ }^{2}{ }^{2}$ A Ato-Chimie (ELF-TOTAL) de Marseille l'ordre est $N \rightarrow M \rightarrow S$ (cf. aussi les travaux de Reinberg).

$\left({ }^{3}\right)$ Des études médicales tendent à montrer qu'il faut prendre $a \geqq 3, b \leqq 7$. Notons que la loi est en passe d'interdire les tours de 7 quarts consécutifs de 8 heures.

$\left({ }^{4}\right)$ Il semble qu'un minimum de 2 jours de repos avant et après les tours de nuits soit médicalement recommandé. 
de jours de repos devant précéder (les $\alpha$ ) et suivre (les $\omega$ ) les tours de $N, S$, $M, J$, on impose donc $\alpha_{i} \geqq 1, \omega_{i} \geqq 1, i=N, S, M, J$. Dans un cycle on peut imposer qu'il y ait au moins un certain nombre de tours de repos dont la longueur soit comprise entre deux bornes fixées a priori.

Les vacances ne sont normalement pas prises en compte dans les cycles. L'habitude est en effet que les opérateurs aient une certaine latitude dans le choix des congés auxquels ils ont droit, ceci étant plus ou moins subordonné d'ailleurs aux diverses disponibilités concernant leur remplacement.

\section{- hypothèses sur le taux de travail hebdomadaire}

$7^{\circ}$ L'horaire hebdomadaire (que nous appelons horaire hebdomadaire moyen), noté $h_{S}$, est fixé. Aujourd'hui on a généralement :

$$
h_{S}=h_{S}^{40}=40 \text { heures par semaine } \quad(S \text { pour semaine })
$$

[cet horaire n'a rien d'universel! (1)].

Pour le travail posté cet horaire n'étant que rarement applicable rigoureusement, on convient d'imposer que le nombre total d'heures de travail dans un cycle divisé par la longueur du cycle en semaine soit égal à $h_{s}$.

Dans le décompte du temps de travail il faut prendre en compte :

- les tours de quarts " normaux " $(N, S, M)$;

- le nombre d'heures de journées normales $\left(h_{J}\right)$;

- éventuellement, le nombre d'heures de passation de consignes dans le cycle.

Nous n'aborderons pas ici les problèmes découlant du calcul des rémunérations correspondant aux heures supplémentaires pour remplacement d'opérateurs absents $\left({ }^{2}\right)$, ni non plus celui du « rattrapage " d'heures ou de journées d'absences.

Pour la généralité, nous admettrons ici que l'on peut tenir compte d'un temps de passage des consignes d'un tour à un autre, à fixer " au mieux » dans une certaine fourchette $[\gamma, \delta]$ (par exemple, $\gamma=5$ minutes, $\delta=10 \mathrm{mi}$ nutes; ou bien $\gamma=\delta=0, \ldots)$.

Pour ne pas trop dérouter le lecteur nous adopterons ici une vue simplifiée des hypothèses $5^{\circ}$ et $6^{\circ}$, à savoir :

\section{- hypothèse}

$8^{\circ}$ Nous n'imposerons de durées minimales de repos qu'après les tours : soient $\omega^{N}, \omega^{S}, \omega^{M}, \omega^{J}$, les valeurs étant toutes au moins égales à 1 .

( ${ }^{1}$ Les syndicats et les ouvriers souhaitent d'ailleurs que cet horaire soit diminué, du moins pour ce qui est du travall par roulement, plus éprouvant pour l'organisme.

$\left.{ }^{2}\right) C f$. à ce sujet les conventions collectives qui varient d'un secteur à un autre. Le dernier document de la C.R.R. contenant ces informations est une note au personnel du 29 août 1974.

$\mathrm{n}^{\circ}$ février 1976. 
Nous allons maintenant exploiter progressivement ces hypothèses de façon à déboucher sur une procédure de génération automatique de roulements de quarts.

\section{3 b. Définition des notations utilisées}

Pour permettre de mieux s'y retrouver nous regroupons ci-après les principales notations que nous utiliserons :

$L \quad=$ longueur du cycle, en jours;

$P \quad=$ nombre d'opérateurs sur un poste;

$P_{0} \quad=$ nombre minimum d'opérateurs sur un poste pour assurer un taux de travail donné;

$n \quad=$ nombre de quarts de nuits (ou de soirs, ou de matins) dans un cycle;

$n_{J}=$ nombre de journées normales dans un cycle;

$n_{J W}=$ nombre de journées de week-ends de repos;

$a, b=$ bornes, inférieure et supérieure, de la longueur d'un tour de nuit, de soirs et de matins. Normalement $a \geqq 3, b \leqq 7$;

$a_{J}, b_{J}=$ bornes, inférieure et supérieure, de la longueur d'un tour de journées normales. Normalement $a_{J}=0, b_{J} \leqq 5$;

$\omega_{N}, \omega_{S}$

$\omega_{M}, \omega_{J}=$ nombre minimal de jours de repos devant suivre les tours de $N, S, M$ et $J$. Normalement on peut raisonnablement prendre $\omega_{N}=\omega_{J}=2, \omega_{S}=1$ ou $2, \omega_{M}=1$ ou 2 suivant que le tour de matins est suivi ou non d'un tour de journées normales;

$h_{s} \quad=$ horaire hebdomadaire moyen;

$\pi=\operatorname{taux}$ horaire moyen $=h_{s} /(24 \times 7)$;

$h_{J}=$ nombre d'heures de journées normales dans un cycle;

$h_{c}=$ nombre d'heures de passation de consignes dans un cycle;

$d \quad=$ durée en heures, du passage des consignes d'un quart au suivant;

$\gamma, \delta=$ bornes, inférieure et supérieure, pour le temps de passage des consignes d'un quart à un autre;

$E^{+}[x]=$ plus petit nombre entier supérieur ou égal à $x$;

$E^{-}[x]=$ plus grand nombre entier inférieur ou égal à $x$.

\section{3 c. Remarques sur le nombre d'opérateurs}

Appelons taux de travail $N S M$, noté $\tau_{N S M}(P)$, le taux de travail en nuit, soir et matin pour un jour pour un nombre d'opérateurs $P$ donné. (Ce taux serait évidemment le même si on raisonnait sur un cycle.) 
Si l'on met $P$ ouvriers sur un poste, on a nécessairement :

Posant :

$$
\tau_{N S M}(P)=\frac{3 \times 8 \text { heures }}{P \times 24 \text { heures }}=\frac{1}{P} .
$$

$$
\pi=\frac{h_{S}}{7 \times 24 \text { heures }} ; \quad \pi_{40}=\frac{40}{7 \times 24 \text { heures }},
$$

on observe que :

$$
\tau_{N S M}(5)<\pi_{40}<\tau_{N S M}(4) .
$$

Théoriquement, si l'on interdit les journées normales et la prise en compte de temps de passage des consignes, il faudrait en fait :

$$
P=\frac{1}{\pi_{40}}=4.2
$$

opérateurs sur un même poste $\left({ }^{1}\right)$.

C'est généralement pour cette raison et parce que $P$ 《 doit être entier » que l'on introduit dans les plannings de roulement des journées normales. On remarquera que les considérations précédentes imposent que $P$ soit plus grand ou égal à un certain nombre $P_{0}$ défini de façon unique, dès que $\pi$ est donné, par :

$$
\tau_{N S M}\left(P_{0}\right) \leqq \pi<\tau_{N S M}\left(P_{0}-1\right) .
$$

On a donc une première relation :

$$
P \geqq P_{0} \text { défini par }(0) .
$$

\section{3 d. Remarques sur la longueur des cycles et le nombre de quarts}

Un nombre $P \geqq P_{0}$ d'opérateurs étant décidé on a, d'après les hypothèses $1^{\circ}, 2^{\circ}$ et $3^{\circ}$ :

$L=$ multiple entier de $7 \times P=k \times(7 \times P), k$ entier $\geqq 1$;

$n=L / P=k \times 7$.

Rappelons que $n$ est le nombre de quarts de nuits dans le cycle d'un opérateur, et que ce nombre est aussi égal au nombre de soirs et au nombre de matins.

En résumé :

$$
L=n \times P \quad \text { et } \quad L=\text { multiple de } 7 .
$$

( $\left.{ }^{1}\right)$ Bien qu'a priori invraisemblable, un taux fractionnaire est réalisable, sans ajouter d'heures supplémentaires (ni en $J$, ni en passage de consigne) lorsque l'on considère la multivalence (cas d'opérateurs appelés «briseurs de quarts » qui alternent d'un poste sur un autre).

n février 1976. 
3 e. Détermination du nombre d'heures de journées normales et de passage des consignes

Le taux horaire moyen $\pi$ étant choisi et une fois choisis les paramètres $P$ et $L$ satisfaisant (1) et (2) on peut déterminer le nombre d'heures de journées normales $h_{J}$ et le nombre d'heures de passage des consignes $h_{c}$, dans un cycle. On doit, en effet, satisfaire un taux de travail hebdomadaire moyen $\pi$. D'où $\left({ }^{1}\right)$ :

$$
\frac{3 \times 8 \times n+h_{J}+h_{C}}{24 \times L}=\pi,
$$

soit :

Nous définirons :

$$
24 n+h_{J}+h_{C}=24 \pi L \text {. }
$$

$$
n_{J}=E^{+}\left[\frac{h_{J}}{8}\right],
$$

où $h_{J}$ est défini par (3) avec :

$$
h_{c}=3 \times n \times d
$$

et

$$
d \in[\gamma, \delta] .
$$

Un moyen simple de calcul de $h_{J}$ et $h_{C}$ est de faire varier $h_{J}$ de 1 en 1 à partir de 0 et de retenir la première valeur telle que :

$$
d=\frac{24 \pi L-24 n-h_{J}}{3 n} \in[\gamma, \delta] .
$$

Il peut bien sûr se faire que l'on ne puisse réaliser (6). Dans ce cas il conviendra de desserrer les bornes $\gamma, \delta$.

Nota : On peut bien sûr modifier la relation (6) afin d'imposer que $h_{J}$ soit un multiple entier de 8 heures. On remplacera (6) par :

$$
d=\frac{24 \pi L-24 n-8 n_{J}}{3 n} \in[\gamma, \delta]
$$

Une fois (6) réalisé, nous sommes dans la situation suivante :

$\pi$ étant donné (taux hebdomadaire moyen);

$P$ étant donné $>P_{0}$;

(1) Le facteur 3 vient de ce que le nombre total de quarts de $N$, $S$ et $M$ est $3 \times n$.

Le facteur 8 vient de ce que les quarts sont de 8 heures (mais la formule reste valable si les quarts sont inégaux et donnent un total de 24 heures sans chevauchement ni temps morts).

Le facteur 24 vient de ce qu'il y a 24 heures dans un « jour »! 
$L$ étant donné $=$ multiple entier de $P$;

on en déduit :

$$
n, \quad n_{J}, h_{c}, d
$$

Nous poussons maintenant l'analyse un peu plus loin.

\section{3 f. Détermination des longueurs des tours}

On sait qu'il y aura $n$ quarts de nuits, $n$ quarts de soirs, $n$ quarts de matins, et $n_{J}$ quarts de journées normales. Il nous faut maintenant déterminer, si c'est possible, des tours dont la longueur soit dans l'intervalle $[a, b]$ pour les $N$, $S$, $M$ et dans l'intervalle $\left[a_{J}, b_{J}\right]$ pour les $J$.

De façon générale le problème se pose comme suit :

- étant donné un nombre entier $n$;

- de combien de manières peut-on décomposer $n$ en un certain nombre $m$ de nombres $n_{i}, i=1, \ldots, m$ tels que :

$$
\begin{gathered}
\sum_{i=1}^{m} n_{i}=n, \\
n_{i} \in[a, b]
\end{gathered}
$$

et quelles sont les valeurs possibles pour $m$ ?

Nous allons indiquer une procédure originale en nous appuyant sur un exemple.

A 1. Recherche d'une première valeur possible de $m$

Prenons $n=21$ et $[a, b]=[4,7]$.

On définit $m$ par :

$$
m=E^{-}\left[\frac{n}{a}\right]=E^{-}\left[\frac{21}{4}\right]=E^{-}[5.25]=5 .
$$

A 2. Recherche d'une première solution réalisable pour $m$ donné

On constitue la suite :

soit ici $\left({ }^{1}\right)$ :

$$
s=\{\underbrace{a, a, \ldots, a}_{(m-1) \text { fois }}, b^{\prime}=n-(m-1) a\},
$$

$$
s=(\underbrace{4,4,4,4}_{4 \text { fois }}, 5),
$$

que nous écrirons pour la commodité :

$$
s=\left(s_{1}, s_{2}, \ldots, s_{m-1}, s_{m}\right) .
$$

(') On notera que l'on a toujours $b^{\prime} \geqq a$.

nº février 1976. 
Dans cette suite on teste si $s_{m} \in[a, b]$ :

- $\mathrm{Si}^{*} s_{m} \notin[a, b]$, on cherche à gauche de $s_{m}$, le premier élément $s_{m-k}(k=1,2, \ldots)$ tel que $s_{m-k}<b$.

S'il n'y en a pas c'est que les bornes $a, b$, doivent être desserrées sinon il n'y a pas (ou plus) de solutions.

On augmente alors $s_{m-k}$ d'une unité et l'on diminue $s_{m}$ d'une unité.

On obtient ainsi une nouvelle suite que nous notons encore :

$$
s=\left(s_{1}, \ldots, s_{m}\right)
$$

- Si $s_{m} \in[a, b]$ on a une première solution du problème $(\star),(\star \star \star)$.

Sinon on reprend le processus tant que nécessaire.

Pour $m$ donné, et une fois en possession d'une première configuration possible $s=\left(s_{1}, \ldots, s_{m}\right)$ on définit toutes les autres solutions de la façon suivante :

\section{A 3. Détermination de toutes les solutions réalisables $s$ pour $m$ fixé}

Pour $m$ fixé, ici $m=5$, nous allons donner un moyen de définir toutes les solutions réalisables de $(\star)$ et $(\star \star)$. Le processus est sans répétition. Il part de $s$ donné par A 2 et définit toutes les autres solutions de proche en proche par le même algorithme. Cet algorithme donne le moyen de passer de la dernière solution déterminée $s$ à une nouvelle solution $s^{\prime}$.

A 3.1. Chercher à partir de la droite du vecteur ligne $s$, et en sautant la dernière composante $s_{m}$, la première composante $s_{m-k}(k=1,2, \ldots)$ telle que :

a) $s_{m-k}<b$;

b) $\exists h \in\{0,1, \ldots, k-1\}$ et tel que $s_{m-h}>a$, et l'on retient le plus petit $h$ tel que cela soit vrai.

S'il n'est pas, ou plus possible, de satisfaire les points $a$ ) et $b$ ) on passe à la phase A 4, sinon on continue en A 3.2.

A 3.2. Augmenter $s_{m-k}$ d'une unité, diminuer $s_{m-k}$ d'une unité.

A 3.3. Retourner le sous-vecteur strictement à droite de $s_{m-k}$ : c'est-à-dire qu'on remplace

$$
s_{m-k+1} \text { par } s_{m}, \quad s_{m-k+2} \text { par } s_{m-1}, \ldots
$$

A 3.4. On a alors une nouvelle solution que nous appelerons encore $s$ et l'on revient au point A 3.1.

\section{A 4. Diminution de $m$}

Une fois obtenues toutes les solutions $s$ pour $m$ fixé, on diminue $m$ d'une unité, on retourne totalement la dernière solution trouvée $\left(s_{1}\right.$ remplacé par $s_{m}, s_{2}$ par $\left.s_{m-1}, \ldots\right)$ et l'on revient en A 2 . 


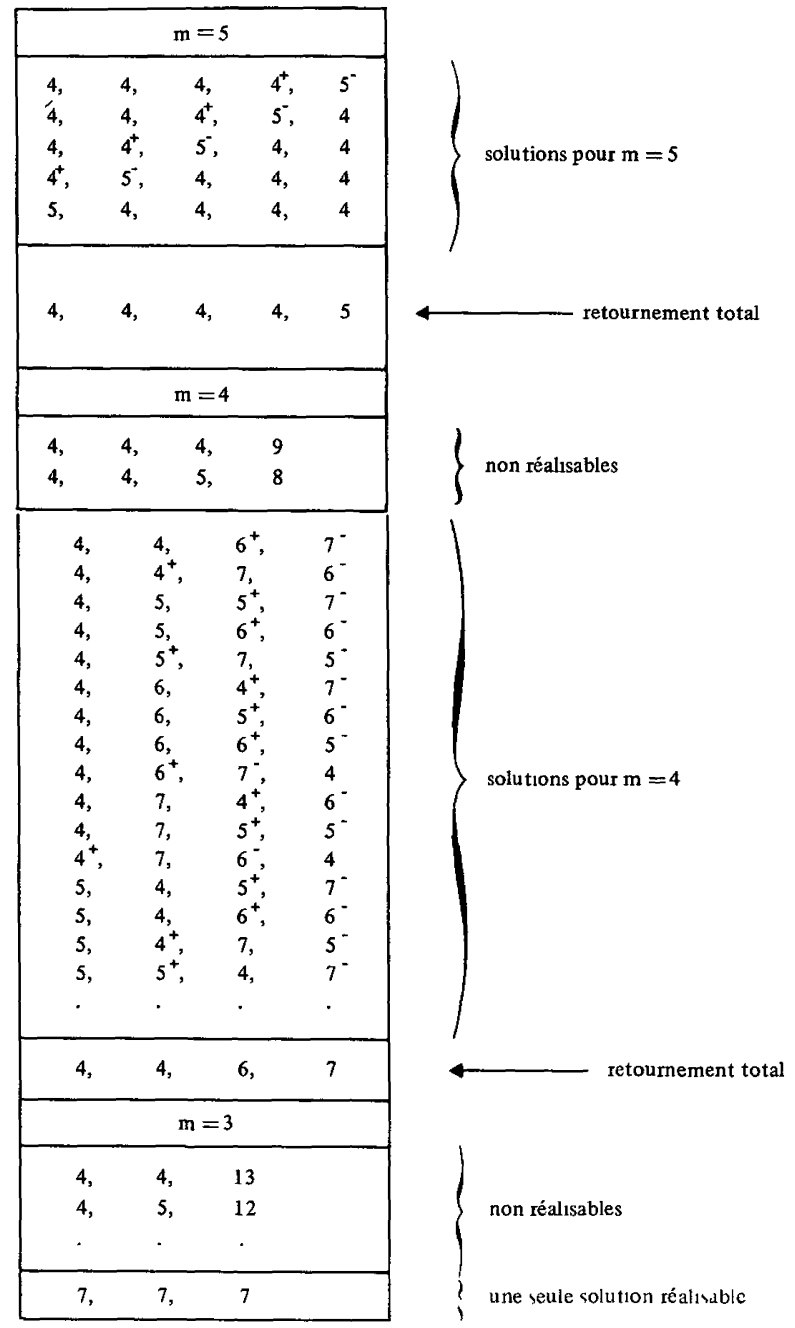

Figure 4.

Pour chaque solution réalisable :

$$
s=\left(n_{1}, n_{2}, \ldots, n_{m}\right),
$$

les composantes du vecteur $s$ indiquent que le cycle peut contenir un tour de longueur $n_{1}$, un de longueur $n_{2}$, etc.

Pour chaque sorte de quart $(N, S, M$ ou $J)$ on peut prendre une solution réalisable quelconque de $(\star)$ et $(\star \star)$ (pour $N, S, M$ il faut cependant que les solutions correspondent à un même $m$ ).

$\mathrm{n}^{\circ}$ février 1976. 
Soient :

$$
s^{N}, s^{M}, \quad s, s^{J},
$$

ces solutions; alors un cycle, a priori possible, contiendra :

$m$ tours de nuits de longueurs respectives $s_{1}^{N}, \ldots, s_{m}^{N}$;

$m$ tours de soirs de longueurs respectives $s_{1}^{S}, \ldots, s_{m}^{S}$;

$m$ tours de matins de longueurs respectives $s_{1}^{M}, \ldots, s_{m}^{M}$;

$m^{\prime}$ tours de journées normales de longueurs respectives $s_{1}^{J}, \ldots, s_{m^{\prime}}^{J}$.

La figure 4 illustre ce qui précède pour $([a, b)]=[4,7]$.

On remarquera que les solutions sont obtenues dans l'ordre lexicographique naturel.

\section{$3 \mathrm{~g}$. Remarques sur le nombre de week-ends}

Il nous a souvent été demandé des cycles contenant le plus possible de week-ends.

Nous allons voir qu'il s'agit là en quelque sorte d'un faux problème!

Du fait de l'égalité des cycles pour tous les opérateurs, et si l'on ne considère pas le problème du positionnement des $J$ (nous parlerons alors de cycle brut), il est assez aisé de voir que dans un cycle le nombre de jours de week-ends, $n_{J W}$, non occupés par des tours de $N, S, M$, est une constante ne dépendant que de $P$ et $L$. On a en effet, du fait de la contrainte de similarité des cycles :

le nombre de lundis occupés par des $N$ est $n / 7$;

le nombre de mardis occupés par des $N$ est $n / 7$;

etc.;

le nombre de lundis occupés par des $S$ est $n / 7$;

le nombre de mardis occupés par des $S$ est $n / 7$;

etc.;

le nombre de lundis occupés par des $M$ est $n / 7$;

le nombre de mardis occupés par des $M$ est $n / 7$;

etc.;

le nombre de lundis occupés par des $R$ (repos) ou des $J$ est $n / 7$;

le nombre de mardis occupés par des $R$ (repos) ou des $J$ est $n / 7$; etc.,

d'où à l'évidence (en interdisant des $J$ pendant les week-ends) :

le nombre de samedis occupés par des $R$ (repos) est $n / 7$;

le nombre de dimanches occupés par des $R$ (repos) est $n / 7$. 
En conséquence, le nombre total de jours de repos tombant un samedi ou un dimanche est une constante ne dépendant que de $P / L$.

D'ailleurs, si l'on définit le taux de jours de week-ends en repos par $\rho=$ nombre de jours de week-ends en repos/nombre total de samedis et de dimanches;

on a :

$\rho=(2 n / 7) /(2 L / 7)$, soit :

$$
\rho=\frac{1}{P} \quad \text { et } \quad n_{J W}=\frac{2 n}{7} .
$$

Autrement dit, le taux de jours de week-ends de repos ne dépend que $d u$ nombre d'opérateurs mis sur un poste.

Cependant la question posée n'est pas tout à fait sans intérêt car une étude plus attentive du problème nous montre qu'on peut chercher à répondre aux deux questions antagonistes suivantes :

- maximiser le nombre de demi-week-ends;

- maximiser le nombre de week-ends complets.

Nous allons voir ceci sur un cycle brut obtenu pour les paramètres :

$$
P=6, \quad L=12 \times 7, \quad[a, b]=[4,6], \quad \omega^{N}=2, \quad \omega^{S}=1, \quad \omega^{M}=2 .
$$

Le cycle brut considéré est le suivant :

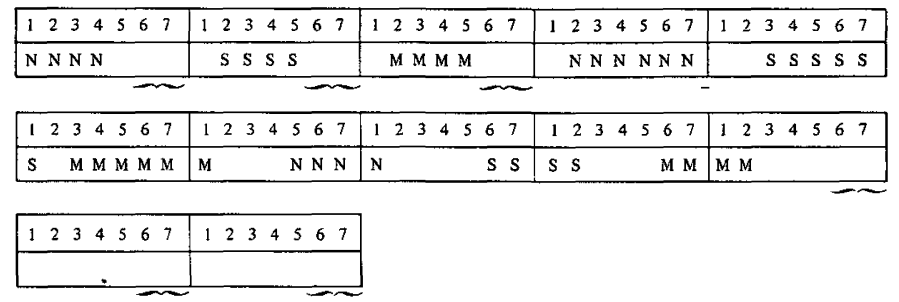

Figure 5 .

On remarquera tout d'abord qu'un cycle brut, donc sans journées normales positionnées, est défini « modulo 7 » c'est-à-dire que « 1 » peut correspondre au lundi, ou au mardi, ... ou au dimanche et que pour chacun de ces choix, il y aura normalement des positionnements différents des tours de $J$ puisque l'emplacement des week-ends dépend de ces choix.

Lorsque « 1 » correspond au lundi on trouve $n_{J W}=2 \times 6=12$ et nous avons le maximum de week-ends complets en repos (6 week-ends).

n février 1976. 
On construit sans difficultés le tableau suivant :

\begin{tabular}{|c|c|c|c|}
\hline lundi en & week-ends en & $\begin{array}{c}\text { nombre de week-ends } \\
\text { complets }\end{array}$ & $\begin{array}{c}\text { nombre de demı } \\
\text { week-ends }\end{array}$ \\
\hline 1 & 6,7 & 6 & 0 \\
\hline 2 & 7,1 & 5 & 2 \\
\hline 3 & 1,2 & 3 & 6 \\
\hline 4 & 2,3 & 4 & 4 \\
\hline 5 & 3,4 & 6 & 0 \\
\hline 6 & 4,5 & 5 & 2 \\
\hline 7 & 5,6 & 4 & 4 \\
\hline
\end{tabular}

Figure 6.

Le choix entre ces diverses solutions n'est évidemment pas aisé surtout quand sur un poste les opérateurs se partagent entre les deux critères.

Les uns voudront par exemple les week-ends en 6,7 ou 3, 4; les autres préférant les week-ends en 1,2 ! On pourra bien sur couper la poire en deux et choisir les week-end en 2,3 ou 5,6 !

\section{3 h. Elimination a priori de certaines configurations}

Avant que d'essayer de positionner les tours, il convient de savoir s'il peut exister des solutions.

En fait, le problème n'est pas si facile car, tant que les tours ne sont pas positionnés, nous ne savons pas quels sont les jours de repos qui correspondront à des week-ends. Malgré cela, nous pouvons déjà faire le test suivant :

Avec $\pi, P, L, n, n_{J}$ donnés par $3 e$ nous vérifierons que l'on peut bien placer a priori $\omega^{N}, \omega^{S}, \omega^{M}, \omega^{J}$ jours de repos après chacun des tours de $N$, de $S$, de $M$, et de $J$. Pour ce faire on regarde si :

$$
3 \times n+n_{J}+m\left(\omega^{N}+\omega^{S}+\omega^{M}\right)+m^{\prime} \omega^{J} \leqq L .
$$

Si ce n'est pas vérifié il faudra diminuer $m$ ou $m^{\prime}$. Si ces diminutions sont impossibles, c'est qu'il n'y a pas de solution et il conviendra :

- soit d'augmenter $L$;

- soit de desserrer les bornes $[a, b]$ et/ou $\left[a_{J}, b_{J}\right]$.

Admettant que (7) est vérifié nous allons maintenant aborder le problème du positionnement des $m^{\prime}$ tours de $J$ puis des $3 m$ tours de $N, S, M$.

\section{3 i. Positionnement des tours de journées normales}

Le problème du positionnement des tours de journées normales est tout à fait différent et beaucoup plus aisé à résoudre que celui du positionnement des tours de $N, S, M$. C'est pour cette raison que nous l'examinerons en premier.

Supposons en effet, que l'on ait pu définir, pour une période $L$, un planning en $N, S, M$. On peut alors positionner les tours de $J$ sans difficulté (s'il 
y a une solution) : en effet il n'est absolument pas imposé dans le planning, qu'il y ait chaque " jour " un ouvrier et un seul en $J$.

Reprenons le cycle brut précédent, et admettons d'abord que « 1 » corresponde au iundi. En imposant $\omega^{N}=2, \omega^{S}=1, \omega^{M}=1$, que les tours de nuits soient précédés de 1 repos, et avec $n_{J}=17\left({ }^{1}\right)$, on obtient :

\begin{tabular}{|c|c|}
\hline $\begin{array}{lllllll}1 & 2 & 3 & 4 & 5 & 6 & 7\end{array}$ & $\begin{array}{lllllll}1 & 2 & 3 & 4 & 5 & 6 & 7\end{array}$ \\
\hline $\mathrm{N} N \mathrm{~N} \mathrm{~N}$ & S S S S \\
\hline$m$ & $\sim$ \\
\hline 12334567 & $\begin{array}{lllllll}1 & 2 & 3 & 4 & 5 & 6 & 7\end{array}$ \\
\hline $\mathrm{M} \mathrm{M} \mathrm{M} \mathrm{M} \mathrm{M}$ & $\mathrm{N} N \mathrm{~N}$ \\
\hline $\begin{array}{llllllll}1 & 2 & 3 & 4 & 5 & 6 & 7\end{array}$ & $\begin{array}{llllllll}1 & 2 & 3 & 4 & 5 & 6 & 7\end{array}$ \\
\hline $\begin{array}{lllll} & \mathbf{J} & \mathbf{J} & \mathbf{J} & \mathbf{J}\end{array}$ & $\begin{array}{lllll} & \mathbf{J} & \mathbf{J} & \mathbf{J} & \mathbf{J}\end{array}$ \\
\hline
\end{tabular}

Figure 7.

On voit immédiatement que nos hypothèses ne permettent pas de positionner les 17 journées normales (on n'en place que 15).

« Décalons » maintenant le planning de façon que « 1 » corresponde au vendredi On obtient :

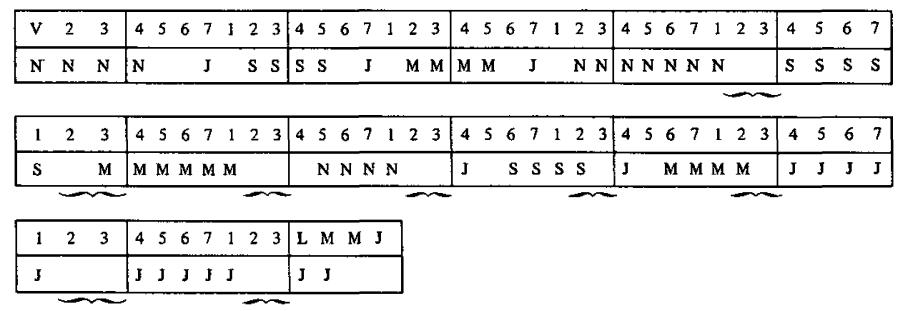

Figure 8.

Dans ce planning on peut positionner les 17 journées normales, et le positionnement est unique si l'on impose que le dernier mercredi soit en repos.

Positionnons maintenant les week-ends en 1, 2. On obtient :

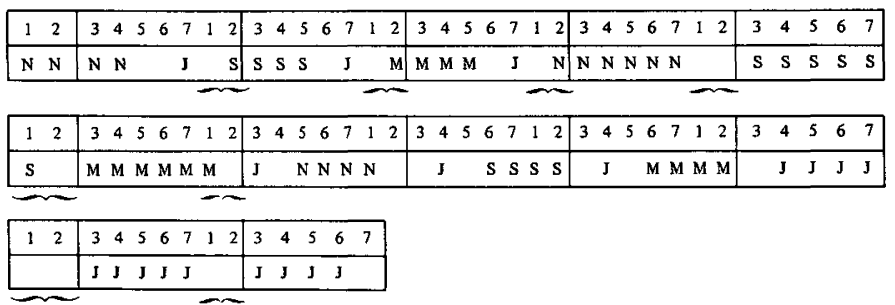

Figure 9.

( ${ }^{1}$ Ce qui correspond à un horaire moyen de $\mathbf{4 0}$ heures avec un temps de passage des consignes de $d=0,190476$ heures, solt presque 12 minutes.

$\mathrm{n}^{\circ}$ février 1976. 
Dans ce nouveau planning on peut placer 19 journées normales soit 2 de plus que le nombre requis.

Pour obtenir un planning définitif correct on pourra retirer 2 journées normales où l'on voudra.

On remarquera que dans aucun des deux plannings " réalisables " qui précédent il n'est possible de satisfaire que les tours de nuits soient tous précédés de deux repos.

Ainsi que nous le voyons ici le positionnement des $J$ ne fait aucune difficulté.

On positionne le lundi de 1 à 7 tant qu'il n'y a pas de solution. Le positionnement des $J$ se fait simplement de la gauche vers la droite en tâchant de satisfaire au mieux des contraintes d'espacement ou de longueurs des tours de $J$. Si cela est possible, c'est-à-dire si l'on a pu positionner un nombre de $J$ au moins égal au nombre requis, toute solution obtenue en retirant l'excédent est acceptable.

Les difficultés que nous avons pu rencontrer jusqu'ici sont cependant négligeables à côté du problème central que nous allons aborder : il nous reste en effet à trouver des cycles bruts réalisables...

\section{LA RECHERCHE DES CYCLES BRUTS}

Le problème central que nous considérons maintenant est le suivant :

- étant donné $P$, nombre d'opérateurs sur un poste;

- étant donné $L$, longueur du cycle en jours;

- étant donné $s^{N}, s^{s}, s^{M}$ (pour un même $m$ ) des longueurs données pour les $m$ tours de $N$, les $m$ tours de $S$, les $m$ tours de $M$;

- définir, si c'est possible, tous les cycles bruts tels que chaque « jour " il n'y ait qu'un et un seul $N$, un et un seul $S$, un et un seul $M$, dans le planning des $\boldsymbol{P}$ opérateurs.

Nous allons résoudre tout cela pas-à-pas.

4 a. Positionnement des débuts des tours d'un cycle brut

En $3 f$ nous avons donné un moyen de déterminer le nombre de tours de $N, S$ ou $M$ ainsi que leurs longueurs possibles.

Un découpage $s^{N}$ étant donné, par exemple :

$$
s^{N}=(4,4,4,4,5), \quad n=21,
$$

on peut imposer le positionnement suivant :

\begin{tabular}{|c|c|c|c|c|}
\hline 1234 & $5 \cdot .1$ & 2345 & 12 & 345 \\
\hline $\mathrm{N} \mathrm{N} \mathrm{N} \mathrm{N}$ & $N N N$ N & $\mathrm{N} N \mathrm{~N} N$ & N N N N & $\mathrm{NNN \textrm {N }}$ \\
\hline
\end{tabular}

Revue Française d'Automatique et Recherche Opérationnelle 
Prenant « 1 » = lundi on a :

le tour $a$ débute un lundi;

le tour $b$ débute un vendredi;

le tour $c$ débute un mardi;

le tour $d$ débute un samedi;

le tour $e$ débute un mercredi.

L'exemple ci-après montre qu'il peut $y$ avoir plusieurs tours débutant un même jour

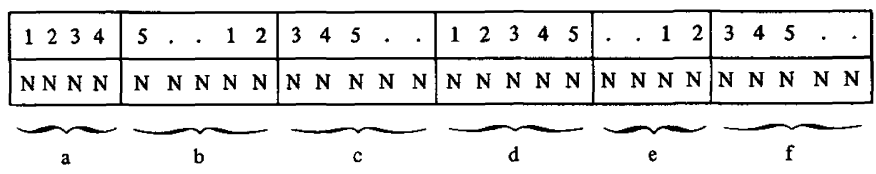

Figure 11.

Ici les tours $a$ et $d$ débutent un même jour et ont des durées différentes, les tours $c$ et $f$ débutent un même jour et ont mêmes longueurs.

On notera que les « jours » de début des tours sont définis modulo 7.

\section{4 b. Ce qu'il ne faut pas faire}

Fort de ces remarques, on peut tenter de définir un cycle en positionnant les tours dans l'ordre :

$$
s_{1}^{N}, \quad s_{1}^{s}, \quad s_{1}^{M} ; \quad s_{2}^{N}, \quad s_{2}^{S}, \quad s_{2}^{M} ;
$$

en respectant les contraintes de repos.

Par exemple avec :

$$
s^{N}=(4,4,6), \quad s^{s}=(4,5,5), \quad s^{M}=(5,4,5) .
$$

\begin{tabular}{|c|c|c|}
\hline (L) & \multicolumn{2}{|c|}{ (Ma) } \\
\hline 1234 & 5.11 & 2345 \\
\hline N N N N & N N N N & N N N N N N \\
\hline (D) & (J) & (Ma) \\
\hline $\begin{array}{llll}1 & 2 & 3 & 4\end{array}$ & $5 \ldots 12$ & $345 \ldots$ \\
\hline s s s s & S S S S S & S S S S S \\
\hline (v) & (Me) & (D) \\
\hline 123345 & $5 . .12$ & 345 \\
\hline M M M M M & \begin{tabular}{l|l} 
M M M M & M
\end{tabular} & \begin{tabular}{l|l}
1 & M M M M
\end{tabular} \\
\hline
\end{tabular}

on a les débuts de tours :

Figure 12.

$\mathrm{n}^{\circ}$ février 1976. 
Avec $\omega^{N}=2, \omega^{S}=1, \omega^{M}=1$ et en calant les tours le plus à gauche possible on obtiendrait le cycle :

\begin{tabular}{|c|c|c|c|c|}
\hline L M M J V . & L M M J V. & L M M J V. & L M M J V . & L M M J V .. \\
\hline$N \mathrm{~N} N \mathrm{~N}-\mathrm{S}$ & S S S - MMM & M M - & $\mathrm{N}-\mathrm{s}$ S S S & $S-M M M M$ \\
\hline L M M J V . & 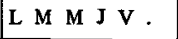 & L M M J V . & L M M J V & L M M J V . . \\
\hline N N N N N N & $-\cdot \cdot-$ & - S S S S S- & $-\cdots-\cdots M$ & M M M M \\
\hline
\end{tabular}

Figure 13.

Mais la question se pose alors de définir le planning général sur 10 semaines des 5 opérateurs sachant qu'ils auraient chacun le cycle défini précédemment.

Nous commençons par positionner le cycle de l'opérateur 1, puis l'opérateur 2 qui «continue » les nuits (noter qu'il peut y avoir ambiguité, si dans le cycle il y a par exemple deux tours de $N$ commençant un même jour qui serait le vendredi). On positionne ensuite l'opérateur 3 ( $c f$. tableau ci-après); malheureusement dans notre exemple il y a une impossibilité résultant d'un chevauchement des soirs :

\begin{tabular}{|c|c|c|c|c|c|}
\hline & LM M J V. & L M M J V . . & L M M J V . & L M M J V.. & $\mathrm{L} M \mathrm{M} \mathrm{JV}$. \\
\hline $\begin{array}{l}1 \\
2 \\
3 \\
4 \\
5\end{array}$ & $\begin{array}{r}\mathrm{NNNN}-\mathrm{S} \\
\mathrm{N} N \mathrm{~N}\end{array}$ & $\begin{array}{r}S \text { S S - M MM } \\
N-\text { - S S S S } \\
\text { N N N N N N }\end{array}$ & $\mid \begin{array}{l}\text { M M - }- \text { N NN } \\
\text { S - M M M M - } \\
\cdots-\ldots\end{array}$ & $\begin{array}{l}\text { N- S S S S } \\
- \text { N N N N N } \\
- \text { S S S S S - } \\
\text { chevauchement } \\
-\end{array}$ & $\begin{array}{l}\text { S - M MM M- } \\
\cdots \cdots \\
\cdots\end{array}$ \\
\hline
\end{tabular}

\begin{tabular}{|l|l|l|l|l|l|}
\hline & L M M J V . & LM M J V . & LM M J V . & LM M J V . . & L M M J V .. \\
\cline { 2 - 6 } 1 & - N N N N N N & $-\ldots-\ldots$ & S S S S S - & $-\ldots$. - M & MM M M - . \\
2 & - S S S S - & $-\ldots .-$ M & MM M M - - & N N N N - S & S S S - MMM \\
3 & & & & & \\
4 & & & & & \\
\hline
\end{tabular}

Figure 14.

Le procédé n'est donc pas bon et force nous est de tenter de définir le planning « globalement».

\section{4 c. Une méthode directe pour la construction des plannings bruts $\left({ }^{1}\right)$}

Nous allons chercher à définir tous les plannings bruts correspondant aux positionnements ( $c f .4 a$ ) résultant d'un choix a priori d'un triplet $s^{N}$, $s^{S}, s^{M}$.

(1) C'est-à-dire sans tenir compte du positionnement des journées normales. 
Pour ne pas compliquer nous ne construirons que les plannings " calés à gauche " c'est-à-dire ceux où les tours de $S$ et $M$ sont le plus à gauche possible. Tous les autres plannings s'en déduiront sans difficulté en considérant tous les décalages possibles vers la droite.

Dans la procédure qui suit nous allons placer dans le planning, d'abord tous les tours de $N$, puis tous les tours de $S$, puis tous les tours de $M$. Disons tout de suite qu'un placement simultané est préférable mais la description du procédé - que le lecteur imaginera aisément - compliquerait inutilement la présentation.

Nous allons donc raisonner d'abord sur les nuits.

Supposant un certain nombre de tours de $N$ positionnés dans le planning de façon qu'il apparaisse 1 et 1 seul $N$ par jour dans le planning depuis le jour 1 jusqu'au jour $j$, et aucun $N$ après $j$, nous appelons « date de positionnement » à ce 'moment le jour $j+1$.

Au départ nous définissons $P$ ensembles identiques $(i=1, \ldots, P)$ :

$E_{i}=$ ensemble des tours définis par $s^{N}$, ces tours étant repérés par leurs longueurs et leurs " jours " de positionnement (modulo 7).

A la date 1 et pour l'opérateur 1 nous pouvons positionner l'un quelconque des tours définis par $s^{N}$. Comme il s'agit d'un problème cyclique nous prendrons par convention le tour $\min \left\{s_{i}^{N}, i=1, \ldots, m\right\}$ et nous le retirons de $\mathrm{E}_{1}$.

A la date $d_{2}=1+\min \left\{s_{i}^{N}\right\}$, nous positionnons à la ligne 2 du planning l'un quelconque des tours de $E_{2}$ qui peut être positionné en $d_{2}$ et nous le retirons de $\mathrm{E}_{2}$.

Le tour choisi ayant une certaine longueur nous avons une nouvelle date de positionnement $d_{3}$. A ce moment on peut a priori positionner un tour de $E_{1}$ sur la ligne 1 ou un tour de $E_{3}$ sur la ligne 3. On classe l'ensemble de ces possibilités et on positionne le premier élément possible. On est alors amené à une date $d_{4}$.

A la date $d_{4}$ nous pouvons positionner un tour sur l'une quelconque des lignes 2,3 , si en $d_{3}$ nous avons positionné un tour à la ligne 1 , ou bien sur les lignes $1,2,4$ si en $d_{3}$ un tour a été placé à la ligne 3 .

On définit l'ensemble des choix possibles, on en retient un, en retirant le tour choisi de l'ensemble correspondant $E_{i}$, puis l'on continue ainsi. Le procédé s'arrête lorsque $d=L$ et que tous les ensembles $E_{i}, i=1, \ldots, P$ sont vides.

Évidemment dans ce procédé on devra tenir compte

$1^{\circ}$ des distances entre les tours qui devront être suffisantes pour laisser la place à un tour de $S$ et un tour de $M$;

$2^{\circ}$ de l'identité des cycles : dès qu'un nouveau tour positionné est lié sur une même ligne à un ou plusieurs tours on reportera le tour en question

$\mathrm{n}^{\circ}$ février 1976. 
sur toutes les autres lignes où des tours avec lesquels il est relié ont déjà été plącé.

On remarquera que dans le procédé il y a constamment des ensembles de tours et de lignes à choisir. Pour obtenir toutes les configurations possibles relevant d'un même $s^{N}$ on " revient " en arrière à partir de la fin jusqu'à la dernière date de positionnement où un nouveau choix est possible. Puis l'on reprend à partir de ce moment en ayant soin de restaurer les ensembles $E_{i}$ et en retirant les tours placés après cette date.

Évidemment on revient aussi en arrière dans le cas où il n'est pas possible possible d'obtenir une configuration admissible.

Une fois qu'un cycle en $N$ est défini, on passe à la définition des cycles en $S$, puis des cycles en $M$, en s'assurant, au prix de certains "décalages ", que l'imbrication de ces trois cycles les uns dans les autres est possible.

Pour obtenir tous les cycles possibles pour un même triplet $s^{N}, s^{S}, s^{M}$, on revient en " arrière " au dernier choix possible, etc. (d'abord pour les $M$, puis pour les $S$, puis pour les $N$ ).

Une fois examiné toutes les possibilités attachées à un triplet $s^{N}, s^{s}, s^{M}$ on définit un autre triplet (en modifiant $s^{M}, \ldots$ puis toutes les modifications ayant été faites pour $s^{M}$, en modifiant $s^{S}$ en restaurant $s^{M}$ à sa valeur de départ, etc.).

L'examen de tous les triplets permet d'obtenir tous les cycles bruts. Ceci se faisant normalement sans répétition si l'on prend soin de ne pas considérer deux vecteurs $s^{M}$ pour lesquels les tours de même longueur auraient les mêmes dates de positionnement.

Il apparaît clairement que le mieux est de placer les $N, S, M$ simultanément et les procédures possibles se déduisent sans difficulté des diverses indications que nous avons données.

Dans la constitution des cycles on peut évidemment tenir compte d'autres contraintes (par exemple, un tour de " repos " d'au moins tant de jours toutes les 4 semaines, un week-end plein toutes les 4 semaines, etc.), ceci permet bien sûr de limiter l'exploration.

\section{4 d. Considérations sur les débuts de cycles}

En examinant plus attentivement le problème on peut observer que dans un planning les "débuts » des cycles des opérateurs ne peuvent se placer n'importe où.

Reportons par exemple sur un graphique tous les " lundis " d'un planning cyclique de longueur $L=10$ semaines, et supposons que les débuts de cycles soieṇt matérialisés par des croix comme ci-après (voir fig. 15).

Comme chaque " jour » il n'y a qu'un seul $N$, on ne peut placer, a priori, de $N$ aux endroits indiqués par un tiret. 


\begin{tabular}{|c|c|c|c|c|c|c|c|c|c|c|c|}
\hline les lundss $\rightarrow$ & 1 & 2 & 3 & 4 & 5 & 6 & 7 & 8 & 9 & 10 \\
\hline \multirow{2}{*}{ opérateurs } & $\left\{\begin{array}{c}1 \\
2\end{array}\right.$ & $\mathrm{X}$ & - & - & & - & & - & & & \\
\hline & - & $\mathrm{X}$ & - & & - & & - & & & \\
\hline & - & - & $\mathrm{X}$ & & - & & - & & & \\
\hline & - & - & - & & $\mathrm{X}$ & & - & & & \\
\hline
\end{tabular}

Figure 15.

Reportant les cycles les uns en dessous des autres, en faisant coïncider leurs débuts, on obtient la figure ci-dessous.

\begin{tabular}{|c|c|c|c|c|c|c|c|c|c|c|}
\hline & 1 & 2 & 3 & 4 & 5 & 6 & 7 & 8 & 9 & 10 \\
\hline 1 & $\mathrm{X}$ & - & - & & - & & - & & & \\
\hline 2 & $\mathrm{X}$ & - & & - & & - & & & & - \\
\hline 3 & $\mathrm{X}$ & & - & & - & & & & - & - \\
\hline 4 & $\mathrm{X}$ & & - & & & & - & - & - & \\
\hline 5 & $\mathrm{X}$ & & & & - & - & - & & - & \\
\hline
\end{tabular}

Figure 16.

Comme les opérateurs ont des cycles identiques on voit qu'avec des débuts de cycles tels que ceux donnés à la figure 15 , le cycle cherché aurait la configuration suivante.

\begin{tabular}{|c|c|c|c|c|c|c|c|c|c|}
\hline 1 & 2 & 3 & 4 & 5 & 6 & 7 & 8 & 9 & 10 \\
\hline $\mathrm{X}$ & - & - & - & - & - & - & - & - & - \\
\hline
\end{tabular}

Figure 17.

Or il y a contradiction car avec 5 opérateurs et un cycle de 10 semaines chaque opérateur doit faire deux lundis en $N$ (il faudrait donc une case vide dans la figure 17).

Les débuts de cycle ne pouvant se placer n'importe où, nous allons donner quelques règles pour la détermination des débuts de cycle.

Nous rappelons et introduisons tout d'abord quelques définitions :

$P=$ nombre d'ouvriers sur un poste;

$L^{S}=$ longueur du cycle en semaine (nous savons que $L^{S}$ doit être un multiple entier de $P$ );

$n^{S}=L^{S / P}$.

Un planning de longueur $l=P d, d$ entier, est dit en décalage régulier de $d$ si les dates de "débuts" des cycles de chacun des $P$ opérateurs peuvent se définir par les "lundis » des semaines

$$
1+(i-1) d, \quad i=1, \ldots, P .
$$

$n^{\circ}$ février 1976. 
THÉORÈME 1 : Un planning brut en décalage régulier $(d)$ de longueur $l=P d$ se reproduit identique à lui-même.

Démonstration : Ce théorème est trivial si les cycles des opérateurs sont de longueur $L^{S}=1$. Dans le cas général, soit un planning de longueur $l=P d$ dans lequel l'opérateur $i(i=1, \ldots, P)$ commence son cycle le lundi de la semaine $1+(i-1) d$. A la date $1+P d$, l'un quelconque des opérateurs doit faire une «nuit ». Supposons que ce soit un opérateur $i>1$. Du fait de l'égalité des cycles il faut introduire alors une "nuit" à la ligne $i-1$ à la date $1+(P-\mathrm{i}) d$, ce qui est impossible puisque l'opérateur $P$ effectue déjà une " nuit " à cette même date. C'est donc que l'opérateur 1 a une nuit à faire en $1+P d$. On en déduit de la même façon que si un opérateur fait un certain quart à une certaine date $t$, il refera ce même quart à la date $t+P d$, d'où le théorème.

La figure qui suit en montre un exemple :

$L^{s}=18, l=6, P=3$.

$X=$ début du cycle d'un opérateur.

\begin{tabular}{|c|c|c|c|c|c|c|c|c|c|c|c|c|c|c|c|c|c|c|}
\hline les lundis $\rightarrow$ & 1 & 2 & 3 & 4 & 5 & 6 & 7 & 8 & 9 & 10 & 11 & 12 & 13 & 14 & 15 & 16 & 17 & 18 \\
\hline & $\mathbf{x}$ & & & & & & . & & & & & & 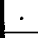 & & & & & \\
\hline \multirow[t]{2}{*}{ opérateurs } & & & $x$ & & & & & & . & & & & & & $\cdot$ & & & \\
\hline & & & & & $\mathrm{X}$ & & & & & & $\cdot$ & & & & & & . & \\
\hline
\end{tabular}

Figure 18.

On déduit de ce théorème le suivant :

THÉORÈME 2 : A partir d'un planning en décalage régulier sur $l=$ diviseur de $L^{S}$ on peut définir de multiples plannings de longueur $L^{S}$, en positionnant pour chaque ligne le début de cycle à l'un quelconque des emplacements où se trouve répété le début du sous-planning.

La démonstration de ce théorème est immédiate en remarquant que le planning d'un opérateur sur une longueur $P d$ se reproduit identique à lui-même (cf. fig. 19).

\begin{tabular}{|c|c|c|c|c|c|c|c|c|c|c|c|c|c|c|c|c|c|}
\hline les lundis $\rightarrow$ & 1 & 2 & 3 & 4 & 5 & 6 & 7 & 8 & 9 & 10 & 11 11 1 & 13 & \begin{tabular}{l|l}
3 & 1 \\
\end{tabular} & 15 & 16 & 17 & 18 \\
\hline \multirow{3}{*}{ opérateurs } & $\mathrm{x}$ & & & & & & $\cdot$ & & & & & . & & & & & \\
\hline & & & $\mathbf{x}$ & & & & & & $\cdot$ & & & & & - & & & \\
\hline & & & & & & & & & & & $\mathrm{x}$ & & & & & . & \\
\hline
\end{tabular}

Figure 19.

Outre les tours que l'on peut définir de cette façon il en est d'autres en particulier dans le cas où $P$ n'est pas un nombre premier ( $c f$. la figure suivante), qui sont en décalages " irréguliers". 


\begin{tabular}{|c|c|c|c|c|c|c|c|c|c|}
\hline les lundis $\rightarrow$ & 1 & 2 & 3 & 4 & 5 & 6 & 7 & 8 \\
\hline opérateurs & $\begin{array}{l}1 \\
2\end{array}$ & $\mathrm{X}$ & & & & & & & \\
\cline { 2 - 11 } & & $\mathrm{X}$ & & $\cdot$ & & & & \\
\hline & & & & & $\mathrm{X}$ & &. & \\
\hline
\end{tabular}

Figure 20.

Nous allons donner maintenant un résultat concernant la détermination de plannings en décalage régulier non redondants.

Soient deux plannings en décalages réguliers $d_{1}$ et $d_{2}, d_{1}>d_{2}$ pour $P$ ouvriers avec $P d_{1}, P d_{2}$ diviseurs de $L^{S}$ :

$$
L^{S}=n_{1} P d_{1}, \quad L^{S}=n_{2} P d_{2} .
$$

Appelons $\pi_{1}$ et $\pi_{2}$ ces deux plannings.

Dans le planning $\pi_{1}$ on trouve des nuits aux dates :

$$
s_{h i}^{(1)}=1+(i-1) d_{1}+h P d_{1}, \quad h=0, \ldots, n_{1}
$$

pour les opérateurs $i, i=1, \ldots, P$.

Dans le planning $\pi_{2}$ on trouve des nuits aux dates :

$$
s_{k j}^{(2)}=1+(j-1) d_{1}+k P d_{2}, \quad k=0, \ldots, n_{2}
$$

pour les opérateurs $j, j=1, \ldots, P$.

Le planning $\pi_{2}$ sera dit redondant si toute configuration que l'on peut obtenir à partir de $\pi_{2}$ peut être obtenue aussi par $\pi_{1}$ ( $c f$. figures ci-après).

On remarque tout d'abord que les deux suites :

$$
\left\{\begin{array}{l}
\left(s_{h i}^{(1)}, h=1, \ldots, n_{1}-1, i=1, \ldots, P\right), \\
\left(s_{k j}^{(2)}, k=1, \ldots, n_{2}-1, j=1, \ldots, P\right),
\end{array}\right.
$$

ont forcément au moins un élément commun. En effet,

$$
n_{1} d_{1}=n_{2} d_{2}=\frac{L^{S}}{P}<L^{S} \quad(\text { pour } P>1)
$$

Or il est clair que les " équations " suivantes :

$$
\left\{\begin{array}{l}
(i-1)+h P=n_{1}, \\
(j-1)+k P=n_{2},
\end{array}\right.
$$

ont toujours des solutions et que l'on a évidemment $h<n_{1}$ et $k<n_{2}$. D'où le résultat.

nº février 1976. 
Soit $\Omega=\left\{t_{r}, r=1, \ldots\right\}$ l'ensemble des éléments communs aux deux suites :

$$
\left\{\begin{array}{l}
\left(s_{h i}^{(1)}, h=0, \ldots, n_{1}-1, i=1, \ldots, P\right), \\
\left(s_{k j}^{(2)}, k=0, \ldots, n_{2}-1, j=1, \ldots, P\right) .
\end{array}\right.
$$

Appelons :

$$
\left\{\begin{array}{l}
i_{r} \text { et } h_{r} \text { les indices } i \text { et } h \text { tels que } s_{h i}^{(1)}=t_{r}, \\
j_{r} \text { et } k_{r} \text { les indices } j \text { et } k \text { tels que } s_{k j}^{(2)}=t_{r}
\end{array}\right.
$$

Si $\forall\left(t_{r}, t_{s}\right), r \neq s$ on a :

$$
\left(i_{r}=i_{s} \text { et } j_{r} \neq j_{s}\right) \quad \text { ou } \quad\left(i_{r} \neq i_{s} \text { et } j_{r}=j_{s}\right),
$$

alors le planning $\pi_{2}$ ne peut être obtenu à partir du planning $\pi_{1}$. En effet, dans ce cas un au moins des opérateurs de $\pi_{2}$ par exemple a au moins 2 « $N$ » à faire tandis que dans $\pi_{1}$ ces 2 « $N$ » sont réparties entre 2 opérateurs différents.

De la proposition précédente on déduit assez aisément le théorème suivant :

THÉORÈME 3 : Si deux plannings en décalages réguliers $\left(d_{1}>d_{2}\right)$ tels que $P d_{1}$ et $P d_{2}$ soient des diviseurs de $L^{S}$ vérifient :

$$
\begin{aligned}
& {\left[P d_{1}, d_{2}\right]^{*} \text { est divisible par } P d_{2},} \\
& {\left[d_{1}, P d_{2}\right]^{*} \text { est divisible par } P d_{1}}
\end{aligned}
$$

(nous notons $[a, b]^{*}$ le p. p. c. m. de a et $b$ ), alors le planning $\left(d_{2}\right)$ est redondant par rapport au planning $\left(d_{1}\right)$.

Si au moins l'une des propriétés (1) et (2) n'est pas vérifiée alors les plannings sont différents.

Nous allons illustrer ce qui précède par quelques exemples :

Exemple 1: Prenons $P=5$ opérateurs, $L^{S}=15$ semaines.

Les plannings en décalage régulier ont les configurations ci-après ( $f g .21 a$ et $21 b$ ).

D'après le théorème 3 , on a, avec $d_{1}=3, d_{2}=1, P=5$ :

$$
\begin{aligned}
{[5 \times 3,1]^{*} } & =15 \text { divisible par } P d_{2}=5 \times 1, \\
{[3,5]^{*} } & =15 \text { divisible par } P d_{1}=5 \times 3,
\end{aligned}
$$

autrement dit le planning de la figure $21 b$ n'est qu'un cas particulier du planning de la figure $21 \mathrm{a}$.

Exemple 2: Prenons $P=5$ opérateurs, $L^{S}=50$ semaines.

Les décalages possibles sont :

$$
d_{1}=10, \quad d_{2}=5, \quad d_{3}=2, \quad d_{4}=1 .
$$




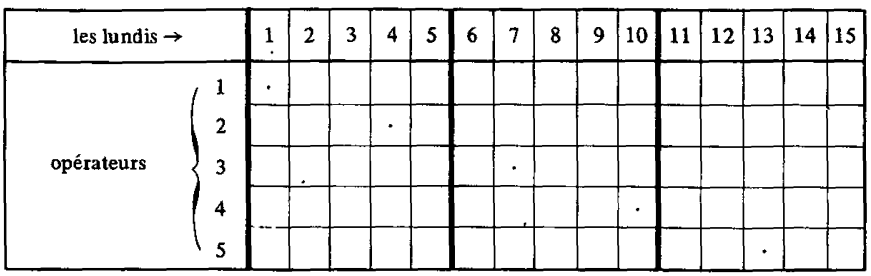

Figure 21 a.

\begin{tabular}{|c|c|c|c|c|c|c|c|c|c|c|c|c|c|c|c|c|}
\hline \multicolumn{2}{|l|}{ les lundis $\rightarrow$} & 1 & 2 & 3 & 4 & 5 & 6 & 7 & 8 & 9 & 10 & 11 & 12 & 13 & 14 & 15 \\
\hline & & & & & & & $\cdot$ & & & & & . & & & & \\
\hline & 2 & & . & & & & & $\cdot$ & & & & & . & & & \\
\hline opérateurs & 3 & & & . & & & & & . & & & & & $\cdot$ & & \\
\hline & 4 & & & & . & & & & & . & & & & & . & \\
\hline & 5 & & & & & - & & & & & & & & & & \\
\hline
\end{tabular}

Figure $21 b$.

On vérifiera par les formules du théorème 3 que les plannings à retenir sont ceux définis par $d_{1}, d_{3}$ et que les plannings $\left(d_{2}\right)$ et $\left(d_{3}\right)$ sont respectivement redondants avec $\left(d_{1}\right)$ et $\left(d_{3}\right)$.

\section{4 e. Intérêt de la détermination des débuts de cycles}

Lorsque l'on définit ainsi a priori un positionnement des débuts de cycles, on peut réduire, souvent de façon considérable les " tâtonnements 》 effectués par la méthode directe. En effet, en se reportant à cette méthode, pour chaque nouveau tour positionné dans le planning on sait immédiatement à quels emplacements dans tous les cycles des opérateurs il convient de le reproduire.

\section{4 f. Exemples de plannings bruts}

Nous allons donner dans ce qui suit des exemples de plannings bruts obtenus par la mise sur ordinateur de la méthode directe dans laquelle on s'est fixé des débuts de cycles régulièrement espacés sur toute la longueur du planning.

Exemple 1: L'exemple qui suit correspond aux desiderata de certaines cellules syndicales :

Il nous a été donné les contraintes et « objectifs 》 suivants :

- 36 heures de travail par semaine (en moyenne);

- pas de temps de passage des consignes;

- 5 ouvriers sur un poste;

n février 1976. 
- 2 jours de repos complet au moins entre les tours;

- tours tous de longueur 5;

- un " minimum " de journées normales;

- un " maximum » de week-ends.

L'ordinateur nous donne alors les informations suivantes :

- les cycles sont d'au moins 25 semaines;

- il y a 7 jours de 8 heures à rajouter plus 4 heures supplémentaires (on compte au total 8 journées normales à rajouter dans le planning brut).

Pour $L^{S}=25$ semaines, il n'y a qu'un seul cycle qui réponde aux conditions du problème.

Le planning obtenu est le suivant :

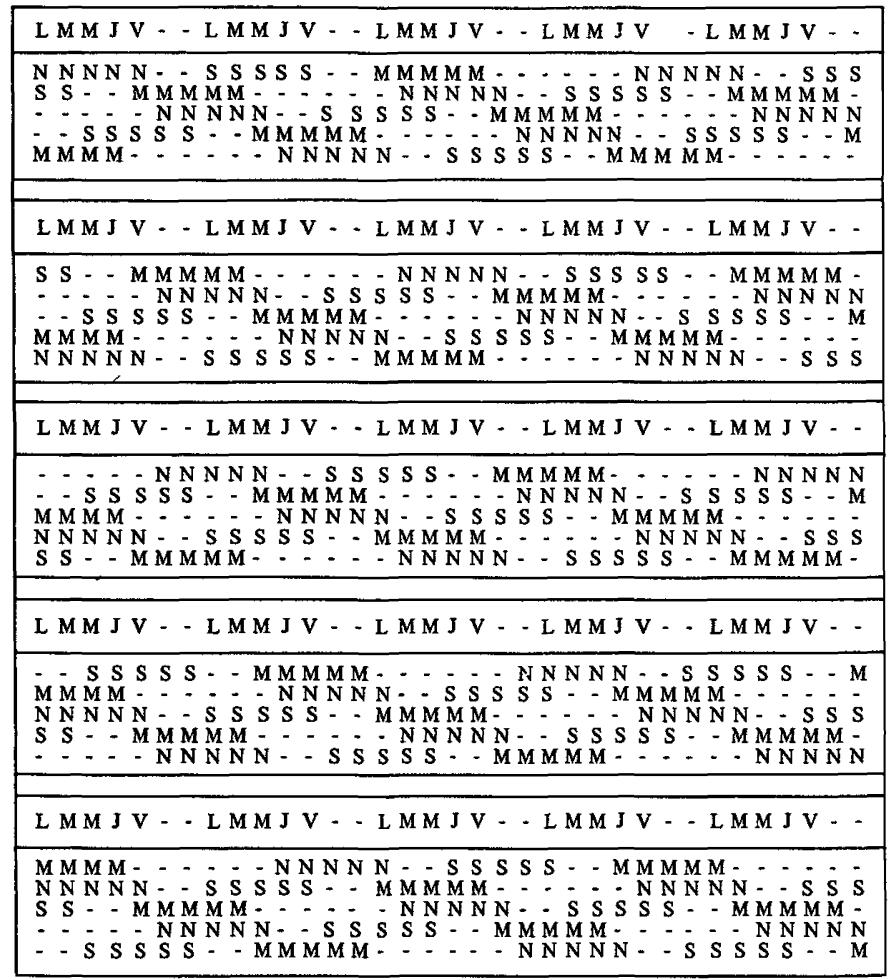

Figure 22.

Exemple 2: Avec

- $P=5, L^{S}=10$ semaines;

- 40 heures de travail en moyenne par semaine; 
- 0 heure de passage de consignes;

- tours de longueur entre 4 et 6 quarts;

- 2 jours de repos au moins entre les tours.

On trouve une centaine de plannings possibles (modulo 7 pour le positionnement du premier jour du planning et modulo le nombre de possibilités de positionnement des journées normales).

A titre d'exemple nous donnons le premier et le dernier planning brut obtenu (il faut rajouter 8 journées normales de 8 heures).

\begin{tabular}{|c|}
\hline$L M M J V--L M M J V--L M M J V-L M M J V-L M M J V--$ \\
\hline 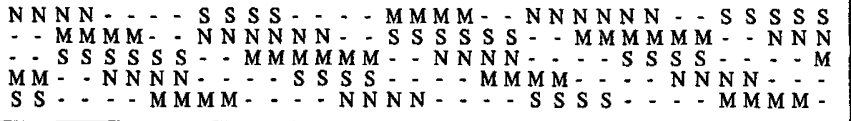 \\
\hline LMM J V - L M M J V - L M J J - - L M J J - - L M J J V - \\
\hline 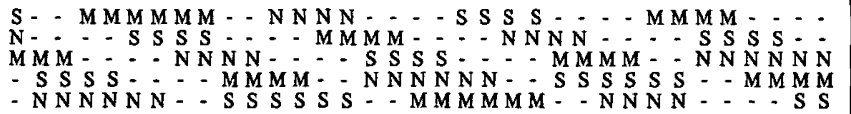 \\
\hline
\end{tabular}

Figure 23.

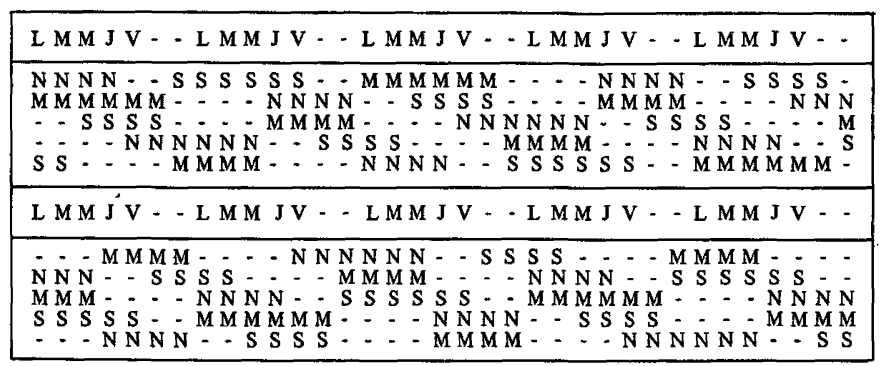

Figure 24.

\section{COMMENTAIRES}

Comme nous l'avons déjà dit plusieurs fois nous sommes loin de pouvoir faire le tour complet d'un tei sujet. La méthode présentée ici permet cependant de s'appliquer à toutes les situations où il s'agit d'un travail en $3 \times 8$ continu en monovalence. Contrairement à ce que l'on pourrait penser, puisqu'il s'agit d'un problème hautement combinatoire $\left({ }^{1}\right)$, la transcription de la méthode sur un calculateur à rapidité moyenne (Télémécanique T 1600) permet d'obtenir

(') A titre d'exemple, avec $L=250$ jours, $Q=5$ ouvriers, il y a a priori $5^{1000}=10^{100}$ possibilités [beaucoup plus que le nombre de protons dans l'univers d'après la célèbre formule d'Eddington $\left(10^{78}\right)$, et le nombre d'êtres vivants ayant existés sur terre, $10^{50}$ d'après G. Salet].

$\mathrm{n}^{\circ}$ février 1976. 
tous les cycles voulus de façon très rapide : l'impression en direct se déroule pratiquement sans attente. D'autre part la recherche et l'impression des cycles de longueur $L$ jours et pour $P$ ouvriers demandent une réservation de mémoire minimale, savoir $L+5 \times P$.

On trouvera de nombreux développements du problème dans des travaux à venir : multivalence $\left({ }^{2}\right)\left({ }^{3}\right)$, polyvalence, gestion des absences, automatisation $\mathrm{du}$ calcul des salaires tenant compte des absences, ...

Avant d'en terminer pour la monovalence nous voulons ouvrir une petite parenthèse au sujet du " casse-tête chinois " de la gestion des congés annuels. D'après la loi, les congés annuels sont pris sur des " jours » normalement retribués.

Autrement dit le travail moyen annuel n'est pas de 40 heures par semaine mais en fait inférieur et d'environ 36 heures.

Supposant que le travail effectif moyen annuel soit ainsi calculé, la « solution " du problème des congés annuels serait de définir des cycles d'au moins 6 mois, sinon de 1 an tels que les congés s'insèrent normalement dans des périodes de repos. Il faudrait ainsi déterminer des cycles tels qu'il y ait environ 3 à 4 semaines de repos complet durant l'été. Chaque ouvrier prendrait ainsi ses congés une année en juin, une année en juillet, une année en août, etc. Ceci résoudrait ainsi le délicat problème de trouver un remplaçant à qui prend 2, 3 semaines de congés, ... ! Mais tout le problème est de savoir dans quelle mesure certains opérateurs pourraient ainsi accepter de prendre leurs vacances par roulement!

\section{REMERCIEMENTS}

Nous tenons à remercier M. Berline, directeur de la Raffinerie de Reichstett, M. Jean, chef du département production, M. Sommelet, ingénieur, M. Kretz, chef du personnel, M. Bietry, chef de la fabrication, M. Baltazar, chef de quart, ainsi que mon ami André Roubaud, opérateur de quart à Shell-Chimie (Berre) pour les passionnantes discussions que nous eûmes avec eux.

Que mon collègue Jean-Louis Le Moigne soit tout particulièrement remercié pour m'avoir mis sur la « piste » de ce curieux problème.

\section{BIBLIOGRAPHIE}

N'ayant pu trouver nul équivalent de cette étude dans la littérature, nous donnons quelques références de problèmes voisins ou bien d'études générales sur le travail par équipes où le lecteur intéressé pourra trouver des indications ou des références utiles.

1. K. BAKer, Scheduling Full-Time and Part-Time Staff to Meet Cyclic Requirements, Operational research Quarterly, vol. $25, \mathrm{n}^{\circ} 1,1974$, p. 65-76.

${ }^{2}$ ) $c f$. J. A. BARtoli et R. Trémolières, Le problème de la multivalence dans le travail continu. Actes du $7^{\mathrm{e}}$ congrès I.F.I.P. sur les Techniques d'Optimisation. (Nice, septembre 1975), Springer-Verlag, 1976.

${ }^{(3)} c f$. J. A. BARtoli, Thèse à paraître, 1976. 
2. K. BAKer, Workforce Allocation in Cyclical Scheduling Problems: Models and Application, Paper No. 122, Graduate School of Business Administration, Duke University, Durham, NC 27706, 1975.

3. C.F.D.T.-F.U.C. (Fédération Unifiée des industries chimiques, I.C.F.), Le travail posté, pour informer, pour agir, automne 1974.

4. W. P. Colquhoun Ed., Biological Rhytms and Human Performance, Academic Press, 1971.

5. M. MAurice, Le travail par équipes, Publications du B.I.T. (Bureau International du Travail), 1971.

6. J. Parsons, Scheduling Two Off Days in a Seven Day Week, Journal of Systems Management, novembre, 1973, p. 42-43.

7. J.M. PLA, Confection automatique de roulements programmes d'agents de conduite, Congrès A.F.C.E.T., Aide à la Décision, t. I, 1974.

8. S. J. Pocock, R. Sergean et P. J. TAYlor, Absence of Continuous Three-Shift Workers, Occupational Psychology, Vol. 46, No. 1, 1972.

9. A. REINBERG, Étude chronobiologique des effets des changements d'horaire de travail (autométrie de 20 sujets postés; système des $3 \times 8$ à rotation hebdomadaire), Archives des Maladies Professionnelles de Médecine du Travail et de Sécurité Sociale (Paris), t. 35, $\mathrm{n}^{\circ}$ 3, mars 1973, p. 373-394 (tirés à part : A .Reinberg, Fondation A. de Rothschild, 29, rue Maurin, 75019 Paris).

10. A. ReInBerg, Les rythmes biologiques, numéro Hors série de Science et Vie consacré à la Biologie, juin-juillet-aout 1974.

11. R. Sergean, Managing Shiftwork, London, Gower Press, 1971.

12. Shiftwork, Incomes Data Study, No. 44, 48, 65, Incomes Data Services Ltd., 140 Great Portland Street, London W.1 01-580 0521/9, 1973. 